\title{
Rail inspection using active thermography to detect rolled-in material
}

\author{
by R. Usamentiaga*, S. Sfarra**, J. Fleuret**, B. Yousefi*** and D. F. García* \\ * Department of Computer Engineering, University of Oviedo, Campus de Viesques, 33204 Gijón, Spain, \\ \{rusamentiaga, dfgarcia\}@uniovi.es \\ ** Department of Industrial and Information Engineering and Economics, Las.E.R. Laboratory, University of \\ L'Aquila, Roio Poggio, Italy, stefano.sfarra@univaq.it \\ *** Computer Vision and System Laboratory, Department of Electrical and Computer Engineering, Laval \\ University, Quebec City, Quebec, Canada, \{julien.fleuret, bardiyausefi\}@gmail.com
}

\begin{abstract}
Non-destructive testing based on infrared thermography has been proven successful in a large number of fields. One area where it has not been fully exploited yet is the inspection of long steel products. Some defects, such as rollingin material, are extremely difficult to detect using dimensional or surface inspection systems. Thus, expensive and complex inspection systems are required. In this work, the feasibility of active infrared thermography for the detection of this type of defects is analysed, where different configurations for the inspection are tested, including power and heating time. Moreover, experiments are applied to real manufactured rails.
\end{abstract}

\section{Introduction}

Rolled steel products are affected by different types of manufacturing conditions that provoke defects visible in the surface of the material or internal [1]. Most of the defects are related to several quality parameters including hardness, strength, formability and dimensions such as width or thickness. Testing for some of these defects requires destructive procedures. In other cases, such as dimensional testing, highly accurate sensors mostly based on optical non-contact technologies are used to measure the dimensions and verify if they are within the expected tolerance for the considered product [2]. Surface inspection systems, also based on optical non-contact technologies such as light projection and image acquisition, are standard equipment in rolling mills in order to detect protrusions or scratches [3]. These inspection systems and quality control in the industry increases the overall quality of the final product and ensures reliability.

There are some defects in steel rolled products that are very difficult to detect using existing inspection systems. Rolled-in material, for example, cannot be detected efficiently by surface inspection systems or by dimensional inspection systems. During manufacturing of a steel product, it passes through several rolls, where the material is compressed, changing the shape in the direction of the least resistance (longitudinal). Rolled-in material defects are created when a part or strip of the rolled product, such as a bar or a rail, is torn off during rolling in a particular roll [4]. As rolling continues, the strip re-attaches to the same product or to a different product manufactured next. Given the high pressure applied during rolling, the rolling-in material re-attaches smoothly to the surface of the product. In most cases, it is very difficult to distinguish it based on the colour, which makes surface inspection systems fail to detect this type of defect. Moreover, this type of defects is critical for the safe long-term operation of the product, as the reattached material is not firmly merged with the rest of the material in the manufacturing product and can create a hole. In this case, the integrity of the material is seriously affected and it could provoke dramatic consequences as it could lead to a reduction in the durability of rail, increasing the probability of derailment. This issue is especially important in rails manufactured for high-speed trains. Consequently, expensive and complex inspection systems are applied to rail manufacturing in order to detect this type of defects early, for example using ultrasonic and eddy current [5]. This way, the defect can be assessed by quality inspectors to determine the required actions. In some cases, the part of the product containing the defect is cut and returned to the steel furnace to be recycled as scrap.

Active thermography has been applied successfully for non-destructive to a large variety of fields, including for example carbon fibre reinforced composites, aluminium, walls or concrete [6]. Infrared thermography is a proven technique with outstanding advantages compared to other non-destructive methods [7], as it is fast, safe and suitable for prolonged and repeated use. New applications are discovered frequently in unrelated fields, for example in physiology [8]. In active thermography, a thermal stimulus is applied to the inspected material. The generated thermal waves travel through the material and find resistances when subsurface anomalies are present. This phenomenon results in thermal contrast on the surface of the inspected material, which can be observed using an infrared camera with adequate thermal sensitivity. Consequently, this technique is used to detect subsurface anomalies that cannot be seen on the surface of the material using cameras that only perceive radiation in the visible spectrum, greatly enhancing the capability to detect defects. The accuracy of this technique depends on many parameters, such as the conductivity of the material, the diffusion, the depth of the defects, the size of the defect and the resolution of the infrared sensor used by the camera, or the energy used to thermally stimulate the material. Thus, it is very difficult to predict the results of active thermography for different types of defects, unless a complete model is used, which is also difficult to create and validate. Consequently, testing new materials 
and defects, and evaluating the feasibility of the technique and most adequate configuration is always an invaluable tool for engineers in charge of quality control in the industry.

This work proposes a new inspection method for rails based on active thermography. In particular, the focus of this work are defects of type rolled-in material, because they are very difficult to detect using conventional inspection systems already installed in industrial installations. The objective of this work is the design of an inexpensive inspection system, fast, easy to deploy and use, and with minimal maintenance. All these properties are requirements nowadays of any industrial inspection system. The proposed method is applied to real rails with real defects to tests the detectability and characterization of the defects. Advanced image post-processing and techniques are applied to the acquired data, and the results are evaluated using the signal-to-noise (SNR) metric.

\section{Spatial resolution}

An important aspect in infrared inspection is the spatial resolution. The size of the defect and the distance from the camera to the object determine the required sensor resolution and the required lens. In general, when the size of a defect is doubled it can be detected in twice as much noise. Therefore, the selection of camera sensor and lens greatly depends on the size of the defects that need to be detected. There is also a trade-off between the spatial resolution and the field of view: as the distance from the camera to the inspected object is reduced, the spatial resolution increases and the field of view is reduced.

In order to analyse the spatial resolution of a camera and lens, the projection of points from the scene to the image sensor needs to be studied. The projection of a 3D point in the scene into a 2D point in the image is described using the pinhole camera model, which can be expressed using (1). The parameters that control this transformation are the focal length of the lens $(f)$, the size of the pixel or detector pitch ( $S_{x}$ and $S_{y}$, equal for most cameras), and the position and orientation of the camera with respect to the object of interest $(R t)$. Other parameters, such as $C_{x}$ and $C_{y}$ are translation coefficients that compensate for displacements of the central pixel. The procedure to estimate these parameters can be found in [9].

$$
\left(\begin{array}{c}
c \\
r \\
1
\end{array}\right)=\left(\begin{array}{cccc}
\frac{f}{S_{x}} & 0 & C_{x} & 0 \\
0 & \frac{f}{S_{y}} & C_{y} & 0 \\
0 & 0 & 1 & 0
\end{array}\right) R t\left(\begin{array}{c}
x^{w} \\
y^{w} \\
z^{w} \\
1
\end{array}\right)
$$

This transformation can be simplified when considering that all the points of interest in the scene lie on the same plane. In this case, the transformation can be written as (2), since $z^{w}=0$.

$$
\left(\begin{array}{c}
c \\
r \\
1
\end{array}\right)=\left(\begin{array}{ccc}
\frac{f}{S_{x}} & 0 & C_{x} \\
0 & \frac{f}{S_{y}} & C_{y} \\
0 & 0 & 1
\end{array}\right)\left(\begin{array}{lll}
r_{11} & r_{12} & t_{x} \\
r_{21} & r_{22} & t_{y} \\
r_{31} & r_{32} & t_{z}
\end{array}\right)\left(\begin{array}{c}
x^{w} \\
y^{w} \\
1
\end{array}\right)
$$

The matrix multiplication in (2) can be written as (3), i.e., a homography or planar projective transformation.

$$
\left(\begin{array}{l}
c \\
r \\
1
\end{array}\right)=H\left(\begin{array}{c}
x^{w} \\
y^{w} \\
1
\end{array}\right)
$$

The transformation in (3) provides an invertible mapping between points and lines on the measurement plane and in the image. Thus, (4) can be used to transform from images coordinates to world points.

$$
\left(\begin{array}{c}
x^{w} \\
y^{w} \\
1
\end{array}\right)=H^{-1}\left(\begin{array}{l}
c \\
r \\
1
\end{array}\right)
$$

The transformation in (4) describes the size of a pixel in the object of interest. Consequently, it provides the spatial resolution based on the features of the camera and lens, and the relative position of camera and object of interest. 
Depending on the MFOV, this value should be multiplied by a constant to obtain the spatial resolution of the camera in order to perform temperature measurements. Generally, due physical and optical limitations a single pixel cannot be used to perform measurements, an area of $3 \times 3$ or $5 \times 5$ pixels is required. The size of this area is described using the MFOV.

Rolled-in material defects can appear in any part of the surface of the rail. However, the most critical part is the head of the rail, as it is the surface in direct contact with the wheel of the rail and thus subject to very high stress. Consequently, the head of the rail requires very high quality and needs to be inspected very carefully. As can be seen in figure 1 , there is a wide variety of rail profiles, and the geometry can be very different. The width of the head in most rails is around $70 \mathrm{~mm}$, but there are some rails where this dimension can reach $150 \mathrm{~mm}$ (rails used for cranes).

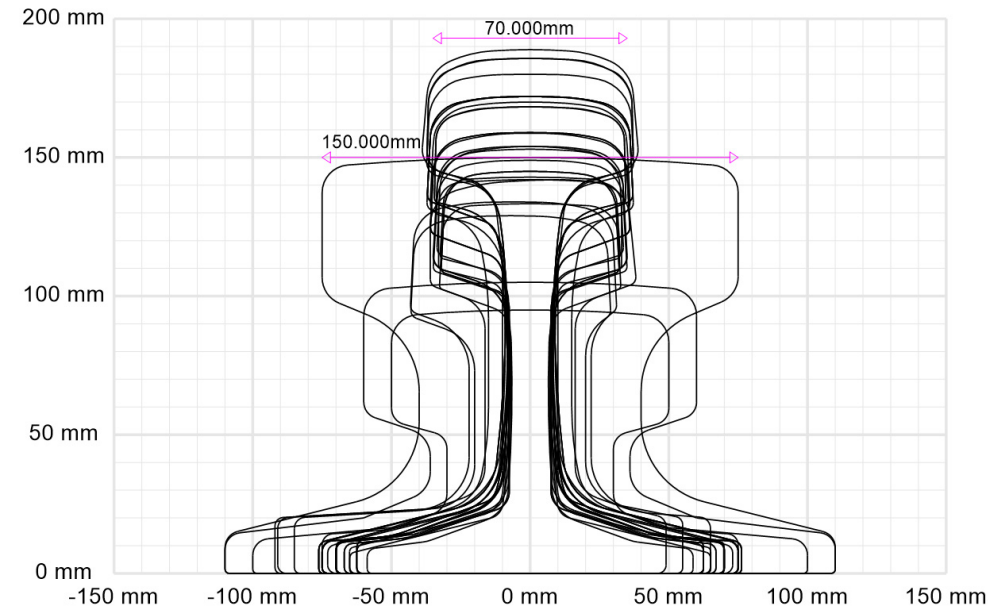

Fig. 1. Geometry of the most common manufactured rails defined in the American and European standards

During manufacturing, rails are rolled and moved forward along a track. A possible location for the inspection system could be after the rolling area, while the rails are moved for final transportation. The infrared camera could be installed in a zenith position, acquiring images from the top of the rail, i.e., the top of the head of the rail. This way, the most important part of the rail is inspected while the rail is being transported. Figure 2 shows two possible configurations of cameras and lens for rail inspection. In the two considered configurations, the camera is installed at 1 meter from the rail, a minimal security distance. The blue parallel lines represent the head of the rail while it is moved, where the transversal axis represents the cross-section of the rail, as can be seen in the figure.

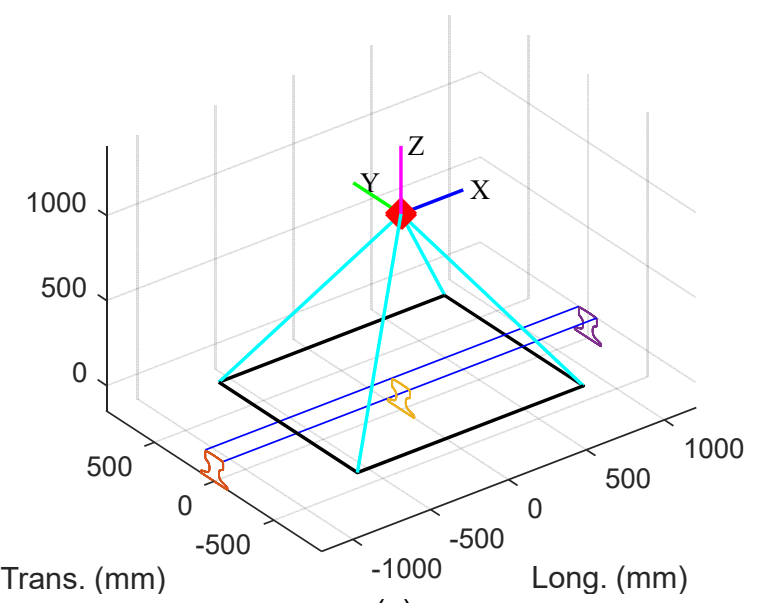

(a)

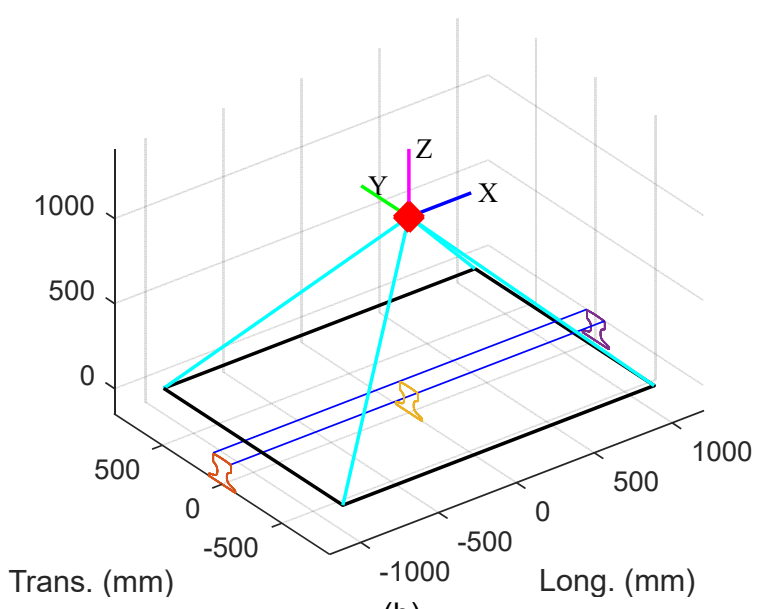

(b)

Fig. 2. Field of view using different camera sensors and lens. (a) Flir A65 with $7.5 \mathrm{~mm}$ lens, (b) Flir A300 with $4 \mathrm{~mm}$ lens

Two different configuration of cameras are lens are considered: the Flir A65 with $7.5 \mathrm{~mm}$ lens and the Flir A300 with $4 \mathrm{~mm}$ lens. In the two cases, lens with the shortest available focal length are selected for each camera, as it is important to maximize the resulting field of view. 
Table 1. Spatial resolution for different cameras and lens when the camera is installed at 1 meters from the rail

\begin{tabular}{lll}
\hline & Flir A65 with 7.5 mm lens & Flir A300 with $4.5 \mathrm{~mm}$ \\
\hline Sensor resolution & $640 \times 512$ & $320 \times 240$ \\
Pixel size & $17 \mu \mathrm{m}$ & $12 \mathrm{um}$ \\
Detector type & Uncooled $\mu$ bolometer & Uncooled $\mu$ bolometer \\
Spectral range & $7.5-13 \mu \mathrm{m}$ & $7.5-13 \mu \mathrm{m}$ \\
HFOV x VFOV (degrees) & $71.91^{\circ} \times 60.25^{\circ}$ & $90.00^{\circ} \times 73.74^{\circ}$ \\
HFOV x VFOV (distance) & $1450.67 \mathrm{~mm} \times 1160.53 \mathrm{~mm}$ & $2000.00 \mathrm{~mm} \times 1500.00 \mathrm{~mm}$ \\
IFOV (distance) & $2.267 \mathrm{~mm} \times 2.267 \mathrm{~mm}$ & $6.250 \mathrm{~mm} \times 6.250 \mathrm{~mm}$ \\
\hline
\end{tabular}

Table 1 shows the spatial resolution when using the two considered configurations. The increased resolution of the Flir A65 provides around three times more spatial resolution at the expense of smaller field of view. The cost of the first configuration is also lower, but less accurate $\left( \pm 2^{\circ} \mathrm{C}\right.$ versus $\left.\pm 5^{\circ} \mathrm{C}\right)$. The thermal sensitivity (NETD) is the same in both cases. Due to the increased spatial resolution and equal thermal sensitivity, the configuration of a Flir A65 with a $7.5 \mathrm{~mm}$ lens is a better choice for the inspection of rails. Based on this configuration, an inspection system could be used to detect defects of $1 \mathrm{~cm}^{2}$, a common size of rolled-in material defects. These defects can be detected in a rail of 1.5 meters in length when the camera is installed at 1 meter of the rail.

\section{Materials and methods}

\subsection{Infrared camera}

The camera used to record the infrared radiation resulting from the thermal stimulus in the experiments is a Flir A65. The most important parameters of the camera can be seen in table 1. This uncooled camera is less sensitive that other cameras that operate in the mid-wavelength infrared band. However, the cost of the uncooled cameras is much lower and the maintenance is greatly reduced. Thus, they are preferred for industrial applications and automation [10].

\subsection{Rail for tests}

The rail used in the experiments is a 136RE rail defined by AREMA (American Railway Engineering and Maintenance-of-Way Association). This type T section rail (flat bottom rail) is made of steel, and it is used for railroad construction. Figure 3 shows an enlarged visible image of the rail with a rolled-in defect. It is important to emphasise that this is not a simulated defect, this is a real rolled-in material defect produced during rail manufacturing.
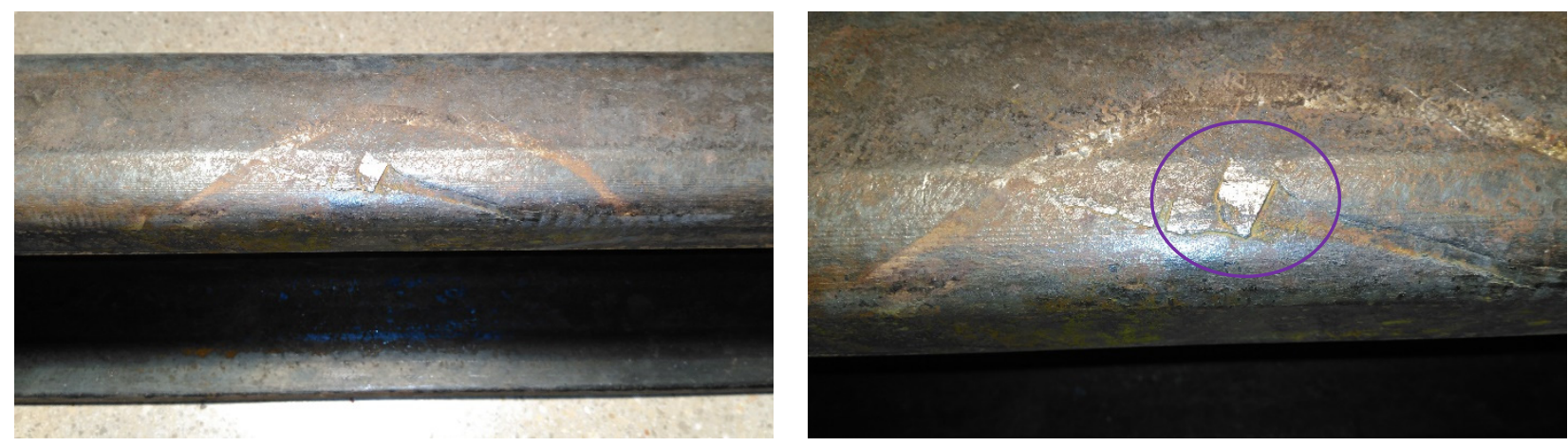

Fig. 3: Image of the test rail in the visible spectrum with a rolled-in material defect.

\subsection{Thermal stimulation}

In the proposed infrared inspection, optical stimulation is applied to thermally stimulate the rail. Two halogen lamps are used, which provide $300 \mathrm{~W}$ each. Therefore, the rail is stimulated with a total of $600 \mathrm{~W}$. Optical step heating is the stimulation method used in the experiments, also referred to as long pulse. The stimulation step can last from a few seconds to a minute, and both the heating and the cooling responses could be of interest. In the experiments performed in this work, the rail is heated for 3 minutes, and only the heating is analysed. An acquisition frequency of $1 \mathrm{~Hz}$ is used in the camera. The experiments are performed in reflection, i.e., the halogen lamps and the infrared camera are on the same side. This is the most appropriate configuration when the defects are close to the surface, which is the case of the rolledin material defects. 


\subsection{Estimation of emissivity}

The emissivity of an object indicates how much radiation an object emits for a specific temperature compared with a black body. For opaque objects, the emissivity and the reflectivity add up to one. Thus, high-emissivity materials have low reflectivity, which makes infrared inspections easier as reflections generate noise in the images.

The emissivity of the rail used for tests has been estimated using the reference emissivity material method. Figure 4 shows an infrared image of the rail during the experiment to estimate the emissivity. As can be seen, there is a piece of electric tape with known emissivity on the rail for temperature reference. The rolled-in material defect can be barely appreciated in the raw infrared images.

The result of the emissivity measurement procedure indicates that the global emissivity of the surface of the rail is 0.91 . Therefore, infrared inspection should not be very affected by reflections.

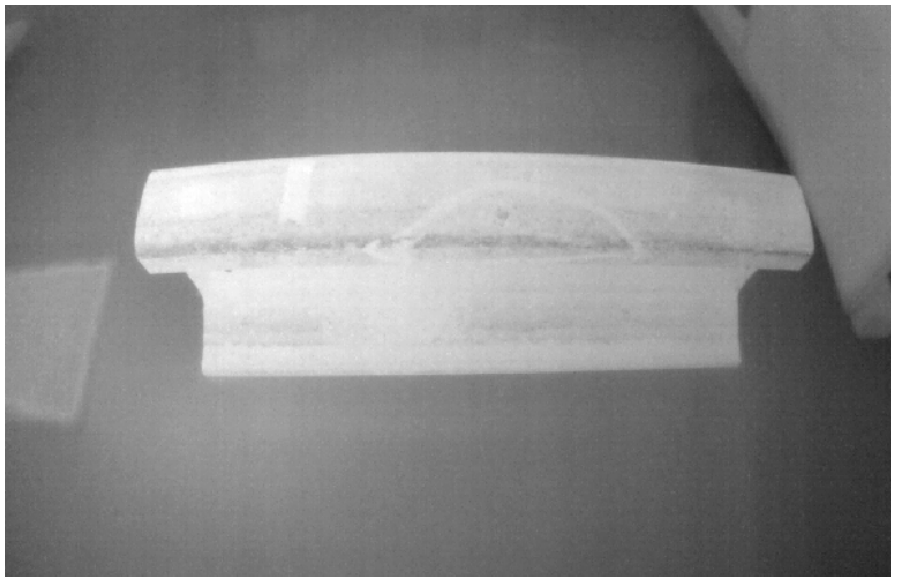

Fig. 4. Infrared image of the rail acquired during the experiment to estimate the emissivity

\subsection{Post-processing}

The results of active thermography show the response of the object to an external thermal stimulus. In order to distinguish defects and anomalies in the inspected object from sound areas, thermal contrast is required. However, this contrast is in some cases very low, and it is lost in the signal noise of the background. Different methods have been proposed to increase the signal-to-noise ratio (SNR) of the defects. Some of these methods are briefly described next.

\subsubsection{Pulsed Phase Thermography}

This method is based on the phase obtained from the Discrete Fourier Transform (DFT) applied to the temperature time history of each pixel [11]. Originally, this method was proposed for active infrared inspections based on optical pulse heating. However, it can also be applied to step heating.

\subsubsection{Statistical moments}

This method calculates statistical moments from the temperature evolution for each pixel, producing a single image from a sequence of frames obtained during heating or cooling [12]. The most common statistical moments used in active thermography are skewness (the third standardized statistical moment) and kurtosis (the fourth standardized statistical moment from a distribution).

\subsubsection{Principal Component Analysis}

This method applies Principal Component Analysis (PCA), a statistical technique of information synthesis, to the temperature evolution of each pixel [13]. The method is thus commonly referred to as Principal Component Thermography (PCT) It also includes a normalization step to improve the results. The first few components (Empirical Orthogonal Functions or EOF) synthesize the temperature evolution, reducing noise in the signal.

\subsubsection{Polynomial fit and derivatives}

This method fits the temperature evolution of pixels to a polynomial [14]. In active infrared inspections based on optical pulse heating, a logarithmic transform before fitting is commonly performed to improve the results. The derivatives of the resulting polynomial and the coefficients of the polynomial are analysed [15]. 


\section{Results}

Figure 5 shows an image of the experiment. As can be seen, the two halogen lamps generate the thermal stimulus and the infrared camera records the resulting infrared radiation.

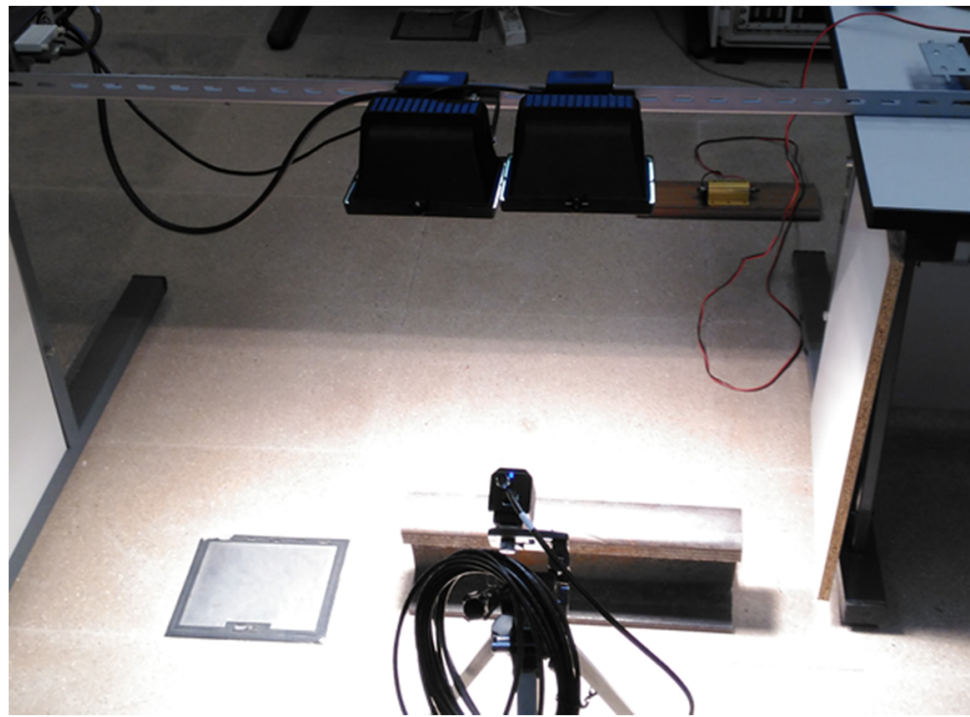

Fig. 5. Inspection of the rail

The sequence of images acquired using the infrared camera is then processed using the described postprocessing methods. Figure 6 shows some of the most representative images of the results. As can be seen, postprocessing methods are not really required in this case, as the rolled-in material defect in the rail can be appreciated in the raw infrared image obtained during heating. Post-processing methods slightly enhance the visualization of the defect, particularly the phase, but they are not necessary to detect the defect. However, they emphasize the defect without needing to enhance the contrast of the images, which is always desired for easier defect detection.

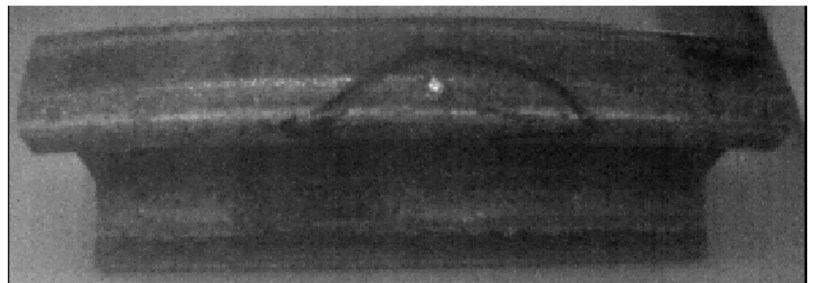

(a)

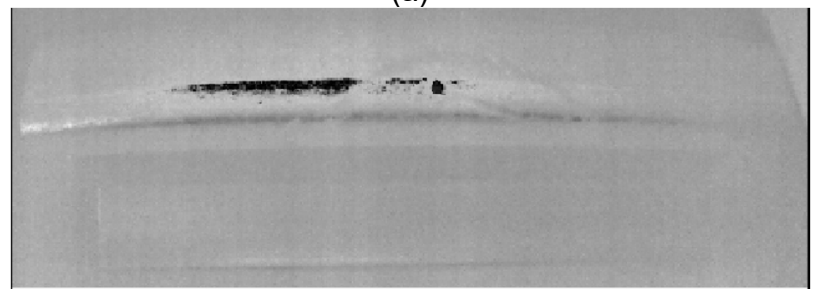

(c)

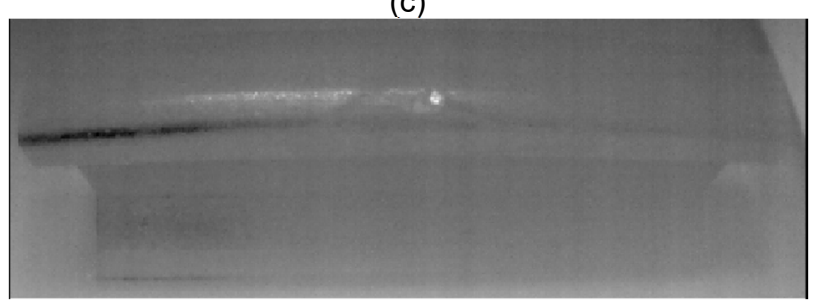

(e)

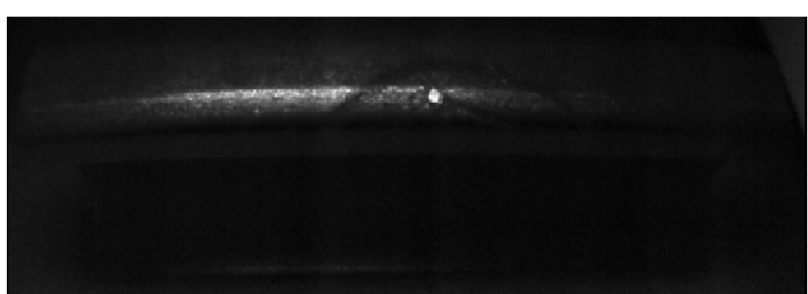

(b)

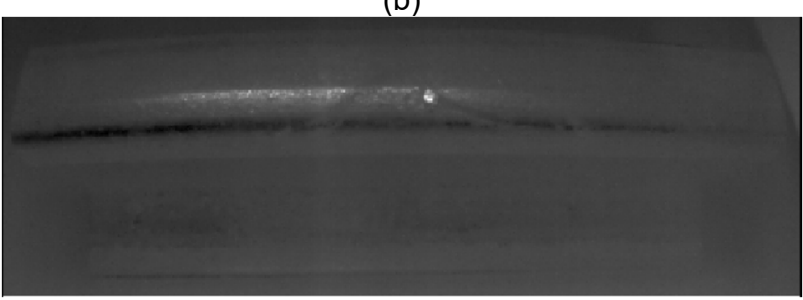

(d)

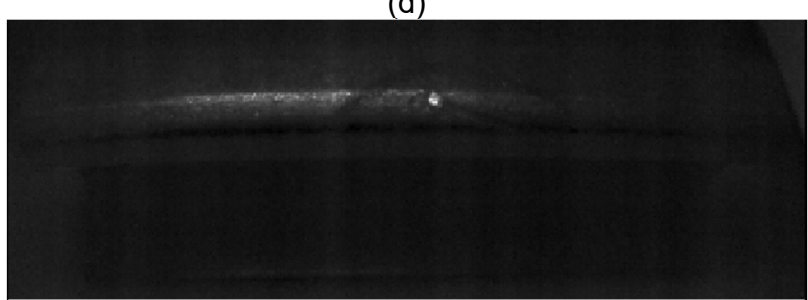

(f)

Fig. 6: Images resulting from the infrared inspection during heating. (a) Raw infrared image, (b) Phase, (c) Kurtosis, (d) PCT EOF2, (e) First derivative of the polynomial fit, (f) Coefficient 5 of the polynomial fit. 
In order to evaluate the results quantitatively, the SNR metric is used to assess the signal-to-noise ratio of the defect. The SNR metric for the defect is calculated using (5), where $\mu_{\mathrm{s}}$ is the arithmetic mean of all the pixels inside the defective area, $\mu_{N}$ is the arithmetic mean of all the pixels inside the sound area, and $\sigma_{N}$ is the standard deviation of the pixels inside the sound area.

$$
S N R=10 \log _{10} \frac{\left|\mu_{S}-\mu_{N}\right|^{2}}{\sigma_{N}^{2}}
$$

The definition of the defective and sound areas has followed the procedure described in [16], using a region around the defect as sound area. The regions are represented in figure 7 . The region in red is the defective area, and the region between the green lines (with dots) is the sound area.

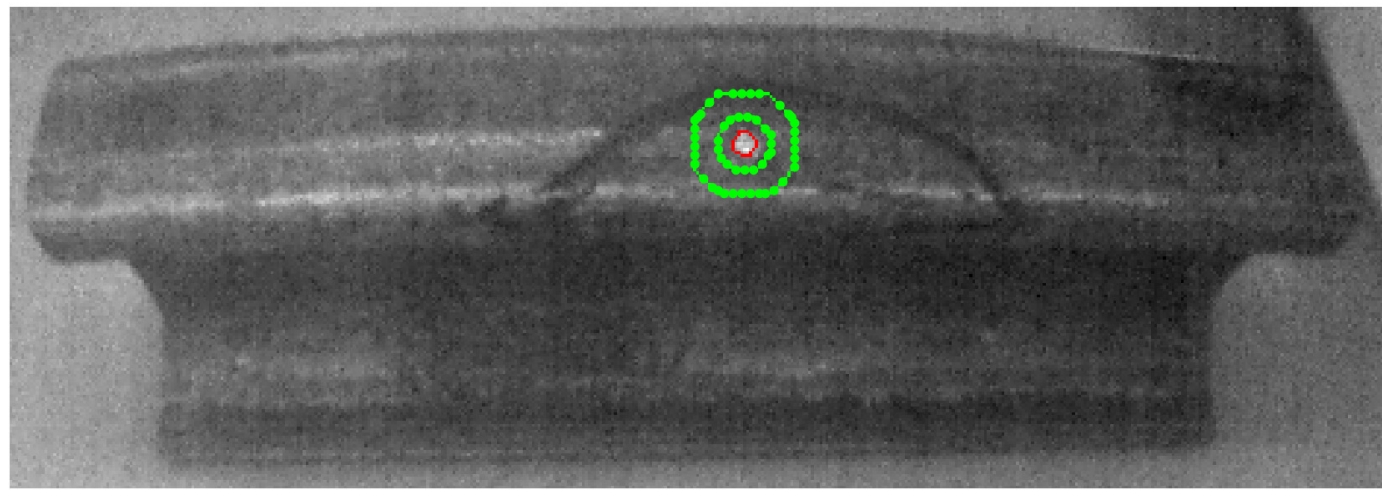

Fig. 7. Defective and sound areas for the quantitative evaluation of the results

The quantitative results are represented in figure 8. Most post-processing methods provide a SNR similar to the raw infrared image. The only exception is the phase, where the SNR is a higher than the other methods. Nevertheless, in this case they do not provide a particular benefit as the defect is clearly appreciated without post-processing, and the raw infrared image provides sufficient thermal contrast.

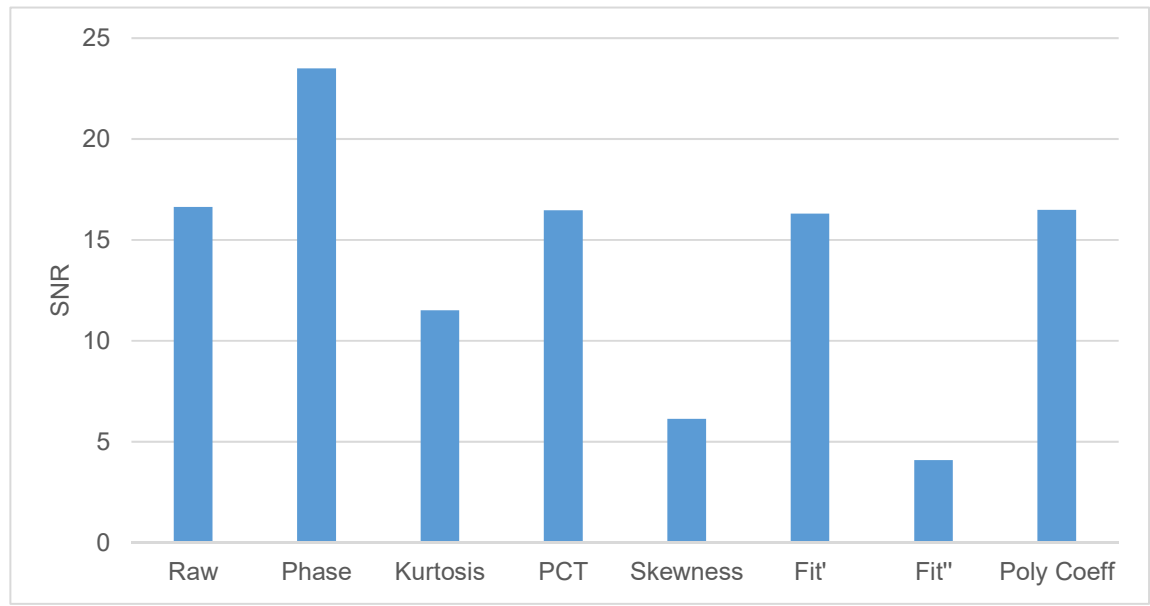

Fig. 8. Quantitative results of the inspection of the rail

\section{Conclusions}

Active infrared thermography is a very useful tool for non-destructive testing in different materials. In this work, it has been applied to rails, where some particular defects known as rolled-in material are difficult to detect using other methods such 3D profile inspection or visual images. These inspection systems are unable to detect these defects, and complex inspection systems based on eddy current are required. In this work, the feasibility of active infrared thermography for the detection of this type of defects is analysed. The results using a real rail indicate that this type of defect can be successfully detected using active thermography. Two halogen lamps have been used to thermally stimulate the rail, and the resulting radiation has been recorded using an infrared camera, where the thermal contrast can be clearly appreciated. Advanced post-processing methods have been applied to the resulting infrared sequence, although the results are not 
significantly improved with respect to the raw infrared images. Further work is necessary to corroborate the results with an extended dataset of defects where the most appropriate heating configuration can be established.

Finally, this work has also studied the spatial resolution obtained with different combinations of infrared camera and lens. Defects of $1 \mathrm{~cm}^{2}$, a common size of rolled-in material defects, can be detected in a rail of 1.5 meters in length when the camera is installed at 1 meter of the rail, providing a safe work distance.

\section{REFERENCES}

[1] Ginzburg VB. High-quality steel rolling: theory and practice. CRC Press; 1993 Feb 23.

[2] R. Usamentiaga, D.F. Garcia, J. delaCalle, "Real-time inspection of long steel products using 3D sensors: calibration and registration", In 2017 IEEE Industry Applications Society Conference, IEEE, vol. 1, pp. 1-8, Cincinnati (USA), 2017.

[3] J. delaCalle, D.F. Garcia, R. Usamentiaga, "Inspection System for Rail Surfaces Using Differential Images", In 2017 IEEE Industry Applications Society Conference, IEEE, vol. 1, pp. 1-8, Cincinnati (USA), 2017.

[4] A.I.S.I. Technical Committee on Rod and Bar Mills, 1996, "Detection, Classification, and Elimination of Rod and Bar Surface Defects", American Steel and Iron Institute, Publication No. MT-003-0.

[5] Thomas HM, Heckel T, Hanspach G. Advantage of a combined ultrasonic and eddy current examination for railway inspection trains. Insight-Non-Destructive Testing and Condition Monitoring. 2007 Jun 1;49(6):341-4.

[6] Maldague, X.P. Nondestructive evaluation of materials by infrared thermography; Springer Science \& Business Media, 2012.

[7] R. Usamentiaga, P. Venegas, J. Guerediaga, L. Vega, J. Molleda, F.G. Bulnes, "Infrared Thermography for Temperature Measurement and Non-Destructive Testing", In Sensors, MDPI, vol. 14, no. 7, pp. 12305-12348, 2014.

[8] Tattersall GJ. Infrared thermography: A non-invasive window into thermal physiology. Comparative Biochemistry and Physiology Part A: Molecular \& Integrative Physiology. 2016 Dec 1;202:78-98.

[9] Usamentiaga, R., et al. "Highly accurate geometric calibration for infrared cameras using inexpensive calibration targets." Measurement 112 (2017): 105-116.

[10] Usamentiaga, R., Ibarra-Castanedo, C., Klein, M., Maldague, X., Peeters, J., \& Sanchez-Beato, A. (2017). Nondestructive evaluation of carbon fiber bicycle frames using infrared thermography. Sensors, 17(11), 2679.

[11] Ibarra-Castanedo, C., \& Maldague, X. (2004). Pulsed phase thermography reviewed. Quantitative Infrared Thermography Journal, 1(1), 47-70.

[12] Madruga, F. J., Ibarra-Castanedo, C., Conde, O. M., López-Higuera, J. M., \& Maldague, X. (2010). Infrared thermography processing based on higher-order statistics. NDT \& E International, 43(8), 661-666.

[13] Rajic, N. (2002). Principal component thermography for flaw contrast enhancement and flaw depth characterisation in composite structures. Composite Structures, 58(4), 521-528.

[14] Shepard, S. M., Lhota, J. R., Rubadeux, B. A., Wang, D., \& Ahmed, T. (2003). Reconstruction and enhancement of active thermographic image sequences. Optical Engineering, 42(5), 1337-1343.

[15] Roche, J. M., Leroy, F. H., \& Balageas, D. L. (2014). Images of thermographic signal reconstruction coefficients: a simple way for rapid and efficient detection of discontinuities. Materials evaluation, 72(1).

[16] Usamentiaga, R., Ibarra-Castanedo, C., \& Maldague, X. (2018). More than Fifty Shades of Grey: Quantitative Characterization of Defects and Interpretation Using SNR and CNR. Journal of Nondestructive Evaluation, 37(2), 25. 